\title{
ORIGINAL ARTICLE Neuromodulation of orexin neurons reduces diet-induced adiposity
}

\author{
AN Zink ${ }^{1}$, PE Bunney ${ }^{2,3}$, AA Holm ${ }^{4}$, CJ Billington ${ }^{4,5,6,7}$ and CM Kotz ${ }^{3,8}$
}

BACKGROUND/OBJECTIVES: Low levels of orexin are associated with obesity and reduced physical activity in humans and animals. SUBJECTS/METHODS: Designer Receptors Exclusively Activated by Designer Drugs (DREADDs) selectively activated orexin neurons in mouse lateral hypothalamus (LH) to measure effects on spontaneous physical activity (SPA). DREADD targeting was achieved by stereotaxic injection of AAV vectors into caudal lateral LH of heterozygous orexin-Cre or C57/B6J mice. In one set of studies, excitation of orexin neurons was examined (virus: AAV2-EF1a-DIO-hM3Dq-mCherry), and test sessions began 3-4 h after light cycle onset. In a study examining the inhibition of orexin neurons (virus: AAV2-hSyn-DIO-hM4Di-mCherry), testing began 15 min prior to dark cycle onset. Clozapine n-oxide (CNO; 1 or $5 \mathrm{mg} / \mathrm{kg}$ ) or saline was injected intraperitoneally and time spent moving in open field chambers was recorded for $2 \mathrm{~h}$. Follow-up studies in separate mouse cohorts quantified SPA in parallel with changes in energy expenditure (EE) and chow intake using indirect calorimetry chambers (SableSystem ${ }^{\mathrm{TM}}$ ). Following acclimation, testing sessions (saline and/or CNO) took place over the course of $\sim 1$ week, with injections administered every day. Changes in SPA, EE, chow intake, fecal boli, and body composition (EchoMRI ${ }^{\mathrm{TM}}$ ) were measured. Additional mice cohorts were fed a high-fat diet (HFD) and injected with CNO daily up to 10 days to assess the potential for orexin activation to prevent diet-induced obesity.

RESULTS: Activation of orexin resulted in increases in SPA in male and female mice, and was accompanied by increases in energy expenditure without changes in overall chow intake. When orexin activation occurred in the context of high fat diet, weight gain and adiposity were significantly attenuated. SPA was decreased when DREADDs were used to inhibit orexin activity.

CONCLUSION: These results demonstrate that orexin neurons play a critical role in mediating physical activity and suggest a novel therapeutic target for treating obesity.

International Journal of Obesity (2018) 42, 737-745; doi:10.1038/ijo.2017.276

\section{INTRODUCTION}

Restoring healthy activity levels throughout the day would have a tremendous impact on the social and medical costs of obesity, ${ }^{1}$ particularly in populations that cannot or do not engage in sustained exercise. The neuropeptide orexin (hypocretin) is a promising therapeutic target for achieving and maintaining healthy body weight. Orexin modulates a number of physiological processes intricately connected to body weight, notably spontaneous physical activity (SPA) and caloric intake..$^{2-5}$ Humans and animals deficient in orexin exhibit reduced physical activity levels and elevated rates of obesity. ${ }^{2,6-8}$ In animals, when orexinergic tone is amplified, either through intracranial peptide infusions or stimulation of orexin neuron activity, increases in SPA and calories burned occurs. ${ }^{3,5,9,10}$ When administered daily for ten days, orexin protects rats against weight gain during high fat diet consumption. 8,11 While these findings are promising, explicit roles of physical activity and energy expenditure (EE) in protecting against adiposity remain undefined, and the specific contribution of endogenous orexin neuron activity under obesogenic conditions is presently unknown.

Orexin is made by neurons located in the lateral hypothalamic area (LH), a highly heterogeneous brain region with mixed cell populations, complex connectivity patterns and functional attributes, ${ }^{10,12}$ including the perifornical and dorsomedial aspects of the hypothalamus ${ }^{13,14}$ as well as in peripheral tissue. ${ }^{15,16}$ Investigations of orexin neuron function in vivo have been limited by an inability to control neural activity in a cell-type dependent manner. Technical advancements in genetics and pharmacology now offer tools to achieve minimally invasive neuromodulation with cellular and anatomic selectivity. A pharmacosynthetic (or chemogenetic) form of neuromodulation, Designer Receptors Exclusively Activated by Designer Drugs (DREADDs) produce sustained alterations in neuronal activity lasting on the order of minutes to hours. DREADDs rely on modified G-protein coupled receptors modified to lose affinity for their biological ligand and gain potent activation by a synthetic designer drug. In this case, the designer drug was Clozapine $\mathrm{N}$-Oxide (CNO), generally thought to be physiologically inert in rodents. ${ }^{17,18}$ Importantly, DREADDs allow for within-subjects experimental designs, preferred for studies measuring dependent variables with known individual variation, like SPA. ${ }^{8,11}$

Cellular and anatomic targeting was achieved in this study by stereotactic injection of a virus containing the DREADD construct, encoded in an inverted open reading frame and flanked by lox-p recombination sites. Orexin cells in LH are theorized to exhibit a medial to lateral functional gradient, such that neurons in the medial portion are more involved in circadian and arousal responses, while lateral areas are more strongly associated with

\footnotetext{
${ }^{1}$ Medical Devices Center, University of Minnesota, Minneapolis, MN, USA; ${ }^{2}$ Minnesota Obesity Prevention Training Program, University of Minnesota, Minneapolis, MN, USA ${ }^{3}$ Geriatric Research Education and Clinical Center, Minneapolis VA Health Care System, Minneapolis, MN, USA; ${ }^{4}$ Food Science and Nutrition, University of Minnesota, Minneapolis, MN, USA; ${ }^{5}$ Department of Medicine, University of Minnesota, Minneapolis, MN, USA; ${ }^{6}$ Minnesota Obesity Center, University of Minnesota, Minneapolis, MN, USA; ${ }^{7}$ Endocrinology section, Minneapolis VA Health Care System, Minneapolis, MN, USA and ${ }^{8}$ Department of Integrative Biology and Physiology, University of Minnesota, Minneapolis, MN, USA. Correspondence: Dr CM Kotz, Department of Integrative Biology and Physiology, University of Minnesota, 2231 6th St. SE, Minneapolis, MN 55455, USA. 
physical activity. To focus on an orexin neuron population thought critical to physical activity, the virus was injected into the caudal lateral $\mathrm{LH}$ of mice engineered to express the DNA-recombinase, Cre, driven by the orexin promoter. The Gq-coupled DREADD, hM3Dq, was chosen for its ability to produce burst-like firing similar to the type of sustained cellular activity observed with neuropeptide release. ${ }^{5}$ A complimentary set of follow-up experiments used the Gi-coupled DREADD, hM4Di, for prolonged neuronal silencing. The systemically administered designer drug, CNO, selectively activated (hM3Dq) or inhibited (hM4Di) DREADDexpressing orexin neurons in caudal lateral LH.

Our manipulations yielded increased activity with increased total energy expenditure, while leaving total caloric intake unchanged. Repeated treatments significantly improved body weight and adiposity outcomes in an obesogenic environment. These results strongly implicate orexin neurons in lateral/caudal LH as promising therapeutic targets for enhancing SPA and energy expenditure and warrant investigation in other metabolic disorders.

\section{MATERIALS AND METHODS}

Animals

Animal use was reviewed and approved by the IACUC of the Minneapolis Veterans Affairs Health Care System (Minneapolis, MN). Adult (8-18 wks) male and female mice, with a C57/B6J background, were maintained on a $12 \mathrm{~h}$ light/dark cycle with chow $(3.0 \mathrm{kcal} / \mathrm{g} ; 14 \%$ calories from fat, $32 \%$ calories from protein, $54 \%$ calories from carbohydrates; Harlan Teklad Rodent Diet) and water ad libitum. Generation and initial phenotyping of orexin-cre heterozygous (orexin-cre) mice and wildtype (Wt) littermates was conducted, and has been described previously. ${ }^{19}$ A subset of male Cre::hM3Dq mice was housed on High Fat Diet high-fat diet $(4.73 \mathrm{kcal} / \mathrm{g}$; $45 \%$ calories from fat, $20 \%$ calories from protein, $35 \%$ calories from carbohydrates; Research Diets, New Brunswick, NJ) for $22 \mathrm{~d}$.

\section{Viral Injections and Drug Administration}

Animals were anesthetized with Ketamine $(70 \mathrm{mg} / \mathrm{kg})$ and Xylazine $(7 \mathrm{mg} /$ $\mathrm{kg}$ ) and placed in a stereotactic apparatus (Kopf Instruments). DREADD targeting was achieved by stereotaxic injection of a Cre-dependent AAV vector expressing double-floxed inverted open reading frame (DIO) around the DREADD transcript and fluorescent tag. Vectors (University of North Carolina Gene Therapy Core) were injected into the LH (AP-1.8/DV-5.0/ML $+/-0.8 \mathrm{~mm}$ from bregma; $333 \mathrm{nl} / 5 \mathrm{~min}$ ) of orexin-cre or Wt mice. Excitatory neuromodulation was achieved via Gq-coupled AAV2-EF1a-DIO-hM3DqmCherry $\left(1.4 \times 10^{12}\right.$ v.u./ml). Inhibition was achieved via Gi-coupled AAV2hSyn-DIO-hM4Di-mCherry $\left(2.0 \times 10^{12}\right.$ v.u./m). Animals recovered from the surgery for at least two weeks prior to testing, and received injections (2/ day) of flunixin $(25 \mathrm{mg} / \mathrm{ml})$ for 3 days' post op.

Clozapine $\mathrm{N}$-oxide (CNO; 1 or $5 \mathrm{mg} / \mathrm{kg}$; $.1 \mathrm{ml} / 20 \mathrm{~g}$ mouse; IP; Enzo Life Sciences, Farmington, New York) or saline vehicle (Veh) was injected via small gauge 3/10CC insulin syringe once per day between 4 and $5 \mathrm{~h}$ postlights-ON (LightCycle) or 15 min before LightsOFF (DarkCycle). Acute tests were followed by a 48-h rest or 'washout' period. To reduce day-to-day variability in acute experiments, data points are reported as the average of two repeated exposures to each test condition. Repeated treatments in the HFD intervention studies consisted of a CNO or Veh injection once per day for 10 consecutive days.

\section{Histology}

Male mice ( $n=3$ /group) were perfused with ice-cold saline and $4 \%$ buffered formaldehyde. For cFos analysis, subjects received an i.p. injection of saline $(n=3)$ or CNO $(n=3), 90$ min before transcardial perfusion. Brains were extracted, post-fixed overnight and then cryoprotected with $~ 10 \%$ sucrose in $3 \%$ formaldehyde (replaced $1 / 3$ of fix with $30 \%$ sucrose solution). Brain sections (40 um, sliced on vibratome) were incubated with blocking solution (0.01M PBS, 3\% Normal Horse Serum; 0.05\% Tween20) for $2 \mathrm{~h}$ at room temp. Slices were transferred to goat anti-orexin antibody (1:1000, Santa Cruz) and rabbit anti-mCherry antibody (1:2000, Abcam) or rabbit anti-cFos antibody (1:2000; Santa Cruz) for $72 \mathrm{~h}$ at $4{ }^{\circ} \mathrm{C}$. The slices were then washed six times ( $20 \mathrm{~min}$ each, at RT) in wash buffer and next incubated with 488-conjugated donkey anti-goat lgG antibody and
Cy3-conjugated donkey anti-rabbit IgG (1:1000, Jackson ImmunoResearch) overnight at $4{ }^{\circ} \mathrm{C}$. Slices were washed five times ( $20 \mathrm{~min}$ each at RT) in wash buffer and one time in $0.01 \mathrm{M}$ PBS before being mounted (GoldBio ProLong/Antifade with DAPI mounting media). Semi quantitative cell counts were collected using the classic visual enumeration technique aided by software algorithms for image processing and counting. Digital images were acquired with a Leica SPE3fluorescent microscope and the Leica Application Suite (LAS 3.3.1 build 8976). Bilateral images were collected from every sixth slice containing the lateral hypothalamus. Using Imaris 8.0 Spot Function software, fluorescent soma and/or nuclei were identified by size ( $12 \mathrm{um}$ and $7 \mathrm{um}$, respectively) and intensity (difference from background). Colocalization was determined by an experimenter and was based on overlapping or adjacent spots. Spots were counted using the internal Spot Function algorithms of the Imaris 8.0. Final data analyses were performed and graphs were rendered in GraphPad Prism 5. Experimenter was blinded to treatment groups for cell counting. Oneway ANOVAs were used to compare the number of cells positive for both mCherry and orexin between orexin-cre ${ }^{+/-}$and wildtype mice, and the number of cells positive for both orexin and cFos between orexin-cre ${ }^{+/-}$ mice that received CNO $(n=3)$ compared to those that received saline $(n=3)$.

\section{Metabolic and behavioral profiling}

Investigations into changes in SPA, or time spent moving (time moving horizontal + rearing) post-injection were initially quantified via infrared beam breaks in three axes, $X+Y+Z$, using MedAssociates ${ }^{\mathrm{TM}}$ Activity Chambers $(17 \times 17$ in). For the orexin neuron excitation study, test sessions started 3-4 h post-LightsON in mice prepared with the excitatory DREADD construct. The time frame was chosen to increase activity at a time when mice are not normally active. Mice were weighed and placed in the activity chambers for $2 \mathrm{~h}$, removed briefly for IP injection and returned to the testing chamber for an additional $4.25 \mathrm{~h}$. At this time, chow pellets were also placed within the activity chambers, which allowed the animals to eat ad libitum during the period of testing. To determine if orexin neuron activity is required for normal physical activity patterns, mice prepared with the inhibitory DREADD construct were given injections of CNO 15 min prior to LightsOFF, and then returned to the testing chambers for an additional $\sim 10 \mathrm{~h}$; chow pellets were also present within the activity chambers during this time. Animals were given at least four days to acclimate to the chambers, and then had an additional three daily injection acclimation sessions, during which saline injections were administered. Testing sessions (saline and/or CNO) took place over the course of $\sim 1$ week, with injections administered every other day. The experimenter was not blinded to these treatment assigments. To eliminate disturbances in SPA caused by handling/injection procedures, data collection started 15 min post-injection. Total chow intake and fecal boli were determined by collecting waste at the end of the $6 \mathrm{~h}$ session.

Follow-up studies investigating changes in SPA alongside variations in EE were assessed using the Mouse Promethion caging system (SableSystem $\left.^{\mathrm{TM}}\right)$. Male mice $(n=6)$ were housed in home-cage conditions with hanging food hoppers filled with chow and water hoppers connected to inverted laboratory balances. An empty plastic tube was suspended from a third laboratory balance and was used to monitor body weight through the test period and Light/Dark cycle. Mice were acclimated to housing conditions for at least $7 \mathrm{~d}$ and habituated to handling and injection procedures for at least $3 \mathrm{~d}$ prior to testing. Test sessions started $4-5 \mathrm{~h}$ postLightsON or $15 \mathrm{~min}$ before LightsOFF, in the activation and inhibition studies, respectively. Data collection started $15 \mathrm{~min}$ post-injection. Spontaneous physical activity, or distance traveled, was quantified via infrared beam breaks in three axes: $X+Y+Z$. Ambient air was passed through the cages $(2 \mathrm{~L} / \mathrm{min})$ and gases were sampled from multiple points within the cage $(250 \mathrm{ml} / \mathrm{min})$. Raw data were collected by SableScreen v2.2 (SableSystem ${ }^{\mathrm{TM}}$ ) every second and extracted using Expedata v1.8.2 (SableSystem ${ }^{\mathrm{TM}}$ ). Indirect calorimetry was calculated using the respiratory quotient, Vmax O2/Vmax CO2, and converted to kCal. The experimenter was not blinded to these treatment assigments.

In a separate set of studies using the Mouse Promethion caging system, the effects of a 10-day treatment regimen of CNO on SPA, EE, and food intake were evaluated in animals fed a high-fat diet (HFD). The experimenter was not blinded to these treatment assigments. Male mice $(n=14)$ were fed a chow diet for 10 consecutive days, while receiving single daily injections of saline. Following this baseline phase, these mice were then placed on a HFD for 22 days, during which time they were injected with either CNO $(5 \mathrm{mg} / \mathrm{kg})$ or saline, once per day, for 10 
consecutive days. The injections of CNO and saline were counterbalanced, with half of the animals receiving the CNO during the first 10 days, and the other half receiving the $\mathrm{CNO}$ injections during the last 10 days. There was a 2-day washout period between phases. Chow and HFD were not counterbalanced within this study, in order to focus on the ability of orexin activation to offset / prevent weight gain when HFD is made available.

Direct measurements of total body fat, lean mass, free water, and total body water were collected from live animals via quantitative magnetic resonance (EchoMRI ${ }^{\mathrm{TM}}$, Houston, TX). Data collection required brief restraint $(\sim 3 \mathrm{~min})$. Reported data are the average of three consecutive scans ( 1 $\mathrm{min} / \mathrm{scan})$ taken $8-9 \mathrm{~h}$ post-LightsON, approximately $26-28 \mathrm{~h}$ following the last treatment. Adiposity index was calculated as the ratio of Fat Mass to Lean Mass. Repeated body composition data collection was separated by 5-10 d. Animals were returned to their home cages for at least $20 \mathrm{~h}$ before subsequent testing ensued.

\section{Statistics}

All statistical comparisons were within-subjects using Repeated Measures analyses. Differences between normally distributed means were evaluated by a one-tailed Student's t-test for two group comparisons. Specifically, all meal pattern analyses for CNO and Saline treatment days were compared using t-tests for the various circadian time points of interest. Parametric one-way and two-way analysis of variance (ANOVA) with the Bonferroni post-hoc correction was performed for other pairwise comparisons among multiple data sets. Data were processed in Microsoft Excel. Statistical analyses and graphing was carried out using GraphPad Prism 5. All data are expressed as mean values \pm S.E.M. A power analysis was used to estimate sample sizes for each experiment. However, due to limitations of the breeding colony, on occasion fewer numbers of animals were used.

\section{RESULTS}

Expression and functional activation of DREADD-mCherry in lateral LH orexin neurons.

Genetic and stereotaxic approaches were used to refine cellular and anatomic targeting of DREADDs, restricting expression to orexin cells in the most caudal and lateral portions of the $\mathrm{LH}$ (Figure $\left.1 ;{ }^{20}\right)$. Adult male orexin-cre (Cre) or wildtype (Wt) mice ( $n=3$ /group) received injections of a DREADD virus ( $h M 3 D q)$ at one site per hemisphere aimed at caudal lateral LH (Figure 1b). Histological LH tissue analysis confirmed selective expression of hM3Dq-mCherry in orexin neurons (Figures $1 \mathrm{c}$ and f). Low magnification images capture nearly the entire orexin neuron field and show clear colocalization between immunoreactive channels labeling orexin (green) and hM3Dq-mCherry (red; Figure 1c). High magnification images show expected cytoplasmic labeling of mCherry and orexin (orange arrowheads, middle row; Figure 1c). mCherry staining was absent in Wt::hM3Dq controls (Figure 1e).

Functional activation of hM3Dq by CNO $(5 \mathrm{mg} / \mathrm{kg})$ in vivo was verified by immunofluorescent labeling of the immediate early gene, cFos (red; Figure 1f). Photomicrographs show cFos staining in both orexin (red arrowheads; Figure 1c) and non-orexin positive cells (white wedges; Figure 1c). High magnification images reflect the expected intracellular distribution patterns with orexin being cytoplasmic and cFos exhibiting nuclear labeling (middle row; Figure 1f). Some endogenous cFos expression was observed in vehicle treated Cre::hM3Dq mice (Figure 1h). Coronal slices of the $\mathrm{LH}$ were imaged at low magnification for visual enumeration (Imaris 8.0; Spot Function) to determine the percentage of infected orexin neurons (Figures $1 \mathrm{~d}-\mathrm{g}$ ). Absolute cell counts and percentages are summarized in Supplementary Materials (Table 1); similar numbers of orexin cells were observed in all genotype and treatment conditions. Our approach infected $69.9 \% \pm 3.2$ of orexin neurons in orexin-cre mice (Figure 1d), which was significantly greater than the $0 \%$ of cells infected in the wildtype mice $(P<0.0001)$. Clozapine $\mathrm{N}$-oxide $(5 \mathrm{mg} / \mathrm{kg})$ induced extensive significant expression of cFos in the $\mathrm{LH}$, and achieved activation in $73.7 \% \pm 2.4$ of orexin labeled cells. The number of cFos immunoreactive cells $(1770.3 \pm 55.8)$ was two to three times greater than the number of orexin neurons observed (565.0 \pm 19.2; $P<0.0001$ ), suggesting the presence of a high degree of excitatory intercellular connections between orexin neurons and other cells in the LH and/or other inputs to the LH. Given the time of day, orexin neurons were expected to be quiescent and exhibit low levels of endogenous cFos $(5.0 \% \pm 0.7)$ in Cre::hM3Dq mice that received a control vehicle (saline) injection.

Changes in SPA, food intake, and EE in male and female mice with access to chow following pharmacosynthetic activation of orexin neurons.

Lateral LH orexin neuron activation during the LightCycle (inactive period) dramatically enhanced SPA in both male $(n=8)$ and female $(n=13)$ Cre::hM3Dq mice (Figure 2a) but not Wt::hM3Dq control males $(n=7)$ and females $(n=8$; Supplementary Fig. $1 A)$. Sex differences in CNO-induced SPA appeared between male and female mice, which were not driven by differences in basal SPA. The sex difference was most dramatic at the lowest dose $(1 \mathrm{mg} /$ $\mathrm{kg}$ ) tested (Figure 2a), which significantly enhanced SPA in females but not males. The largest CNO dose tested $(5 \mathrm{mg} / \mathrm{kg}$ ) significantly enhanced SPA to a similar degree in both sexes (Figure 2a), without producing any significant changes in food intake or fecal boli measurements (Figures $1 \mathrm{~b}$ and c). For this reason, $5 \mathrm{mg} / \mathrm{kg}$ was selected for subsequent experiments.

When tested during the light (inactive) period, acute pharmacosynthetic activation of orexin neurons results in a sustained, reversible, and dose-related effect on SPA (Figure 2a). CNO had no effect on total calories of chow consumed or gastric motility, as measured by number of fecal boli passed during the test session (Figures $2 b$ and $c$ ). The size and duration of effects on physical activity levels were comparable to existing reports, despite slight differences in methodological approach: specifically, a more lateral injection site and a smaller infusion volume (Inutsuka et al., 2014). The metabolic impact of enhanced SPA was investigated further under home-cage conditions (SableSystem; Reno, NV), which enabled quantification of energy expenditure (EE; Figure 3) and other metabolically relevant behaviors (Figure 4). Spontaneous physical activity and total EE were significantly increased in the $24 \mathrm{~h}$ following pharmacosynthetic activation (Figure 3b). Higher resolution analysis identified increased EE during physical activity (ActiveEE) and a slight but not significant increase in EE during inactivity (RestEE; Figure 3b). All of the $24 \mathrm{~h}$ effects were driven by alterations that were restricted to the $6 \mathrm{~h}$ period following CNO treatment (Figure $3 \mathrm{c}$ ). Acute stimulation of orexin neurons in the lateral LH did not impact body weight (Figure 3).

Injections of CNO did not produce cumulative food intake changes over the treatment interval (Figure 4a), but the pattern of intake was altered in the $6 \mathrm{~h}$ period following CNO treatment (Figure 4b). The data show a complementary pattern of an increased number of bouts along with a reduction in the frequency of trips (bouts) to the food hopper. This resulted in a reduction in the amount of food consumed per bout, but no difference in the net intake over this $6 \mathrm{~h}$ period (Figure $4 \mathrm{~b}$ ). The pattern of consummatory behaviors is consistent with enhanced SPA, as seen in Figure 3. Previous work in our and others laboratories has shown that cumulative food intake following stimulation of orexin pathways is not significantly influenced for more than $2 \mathrm{~h}$. Typically, any elevations in acute intake are followed by compensatory feeding reductions in the later hours, yielding a net effect of zero change in food intake over a $24 \mathrm{~h}$ time frame. ${ }^{3,5}$

Effects of repeated stimulation of orexin neurons, during access to high-fat diet, on adiposity, weight gain, food intake, SPA, and EE Adiposity was reduced in adult Cre::hM3Dq male mice $(n=14)$ fed a high fat diet (HFD; 45\%kcal from fat; Research Diets) when given in conjunction with 10 consecutive daily CNO treatments. Treated 
animals had elevated SPA and EE, and gained approximately half as many grams (CNO, $1.4 \pm 0.3$; HFD+Veh, $2.6 \pm 0.4$ ) of body weight (Figure $5 \mathrm{a}$ ). Figure $5 \mathrm{a}$ shows the body weight on the final day of the Chow+Veh phase, indicated by an open, white, circle at $\mathrm{d} 0$, and the differences in weight gain in animals on HFD+Veh and $\mathrm{HFD}+\mathrm{CNO}$ across the 10 days of treatment. Body composition a

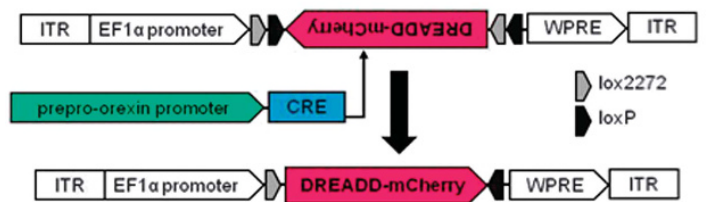

C
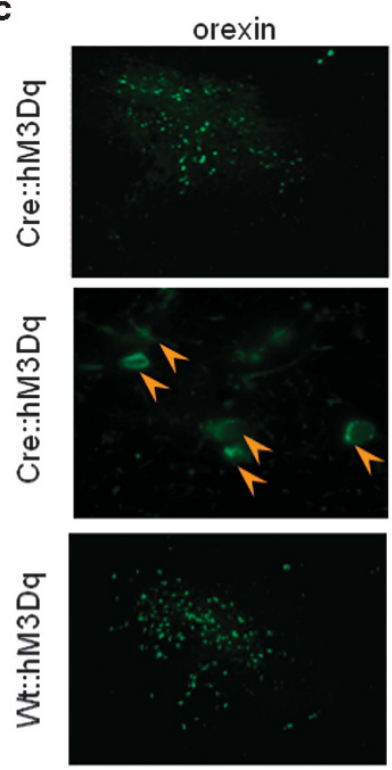

f
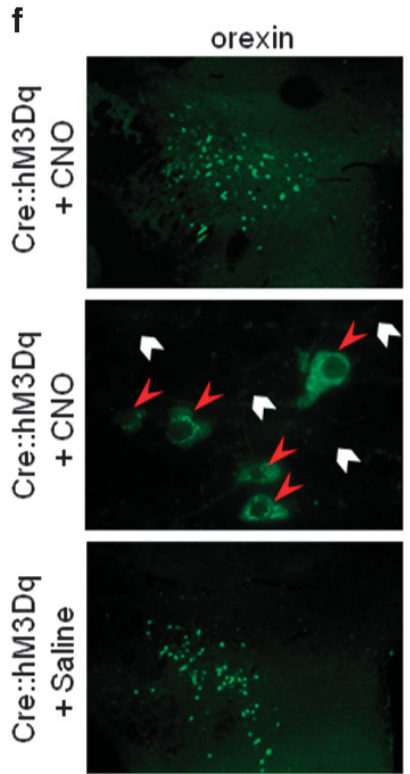

mCherry
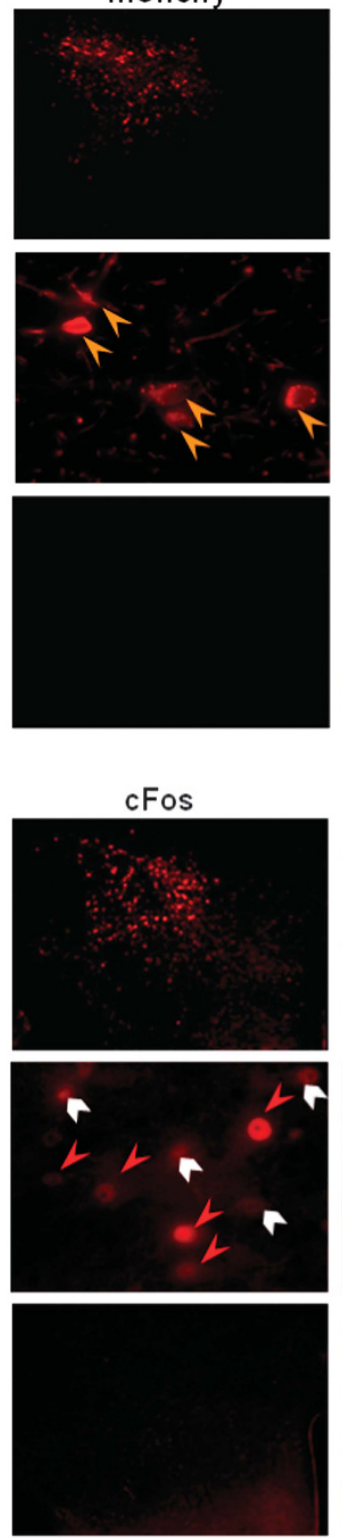

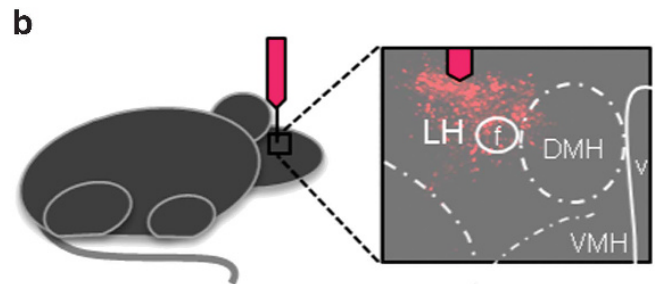

merged
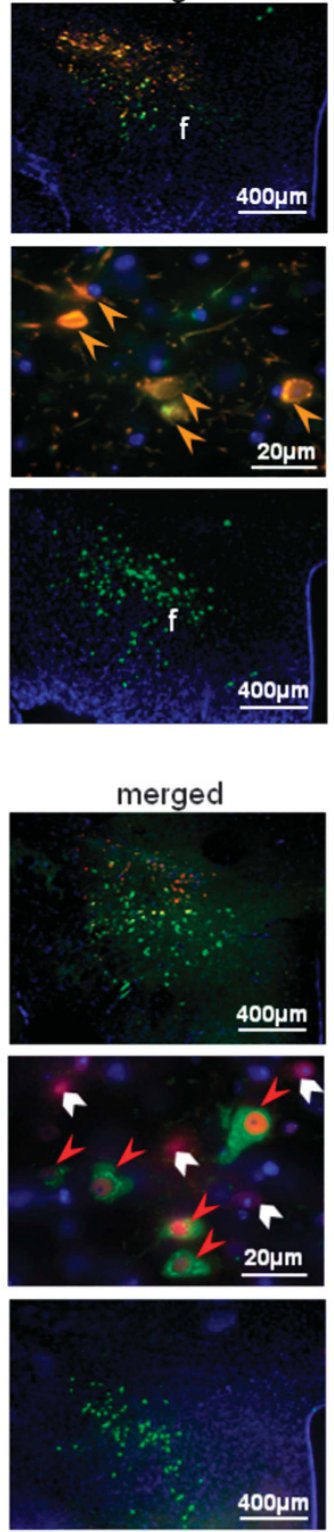

d
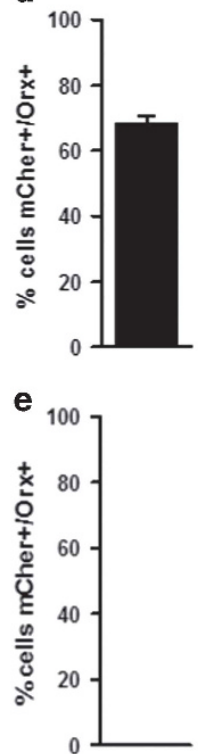

g

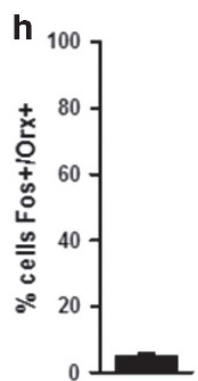

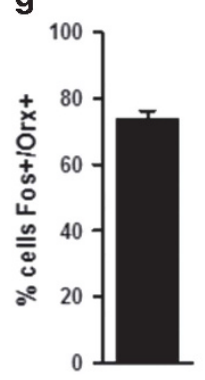

Figure 1. Cre-dependent genetic targeting and pharmacosynthetic activation of orexin neurons $(\mathrm{N}=9)$. (a) Schematic diagram of AAV vector encoding DREADD-mCherry driven by Elongation factor alpha (EFa1) promoter sequence and flanked by dual flox sites for recombination in the presence of Cre-recombinase. Cre is driven by the prepro-orexin-promoter of Orexin:Cre mice. (b) Schematic of anatomical targeting with stereotactic viral infusion. Photomicrographs of coronal sections containing immunofluorescent orexin neurons (green) and hM3Dq-mCherry (red; C) or cFos (red; F). Percent of orexin neurons expressing mCherry (orange arrow head) in Cre::hM3Dq (d) and Wt::hM3Dq (e) mice. Percentage of orexin neurons expressing Fos (red arrowhead) after CNO ( $5 \mathrm{mg} / \mathrm{kg} ; \mathrm{G})$ or vehicle (E). DAPI (blue), nuclear label; Cre, orexin-cre; lateral hypothalamus (LH), fornix (f), dorsal medial hypothalamus (DMH), ventral medial hypothalamus (VMH); inverted terminal repeat (ITR); woodchuck post-regulatory regulatory element (WPRE). 

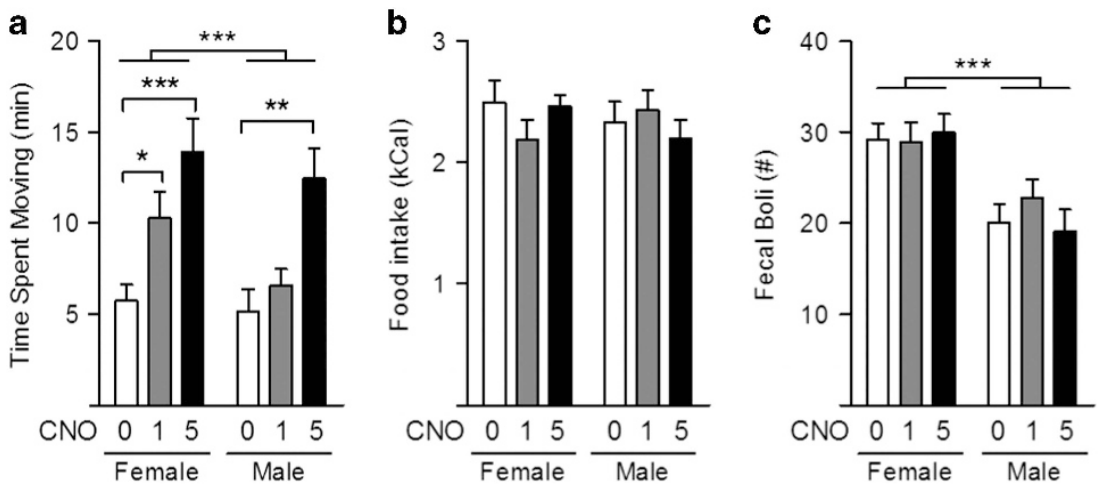

Figure 2. Acute pharmacosynthetic activation of orexin neurons increases SPA in female and male Cre::hM3Dq mice ( $\mathrm{N}=21)$. Behavioral activity in large activity chambers (MedAssociates; St. Albans, VT) $4 \mathrm{~h}$ after an injection of CNO (5 mg/kg; black bars) or vehicle (0 mg/kg; saline; white bars), $5 \mathrm{~h}$ into the Light Cycle. Time spent moving (a), chow intake (b), and fecal boli $(\mathbf{c})$ in female $(n=13)$ and male $(n=8)$ mice. ${ }^{*} P<0.05,{ }^{* *} P<0.01,{ }^{* * *} P<0.001$.

a

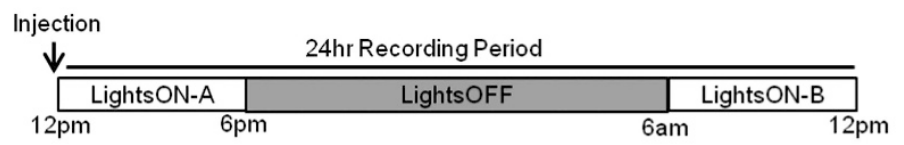

SPA
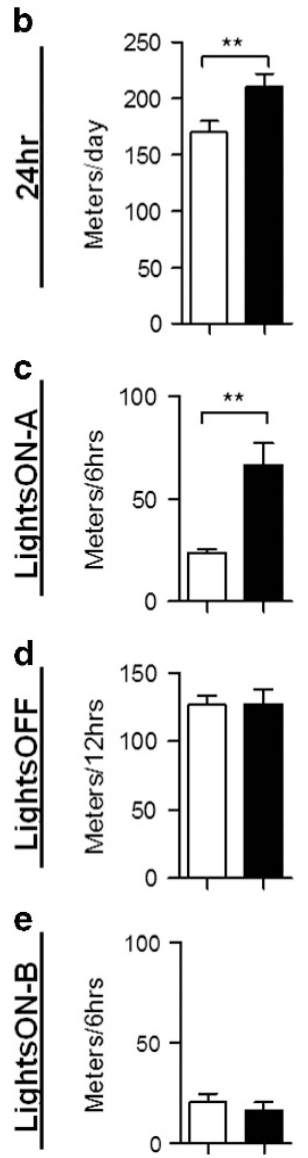

TotalEE
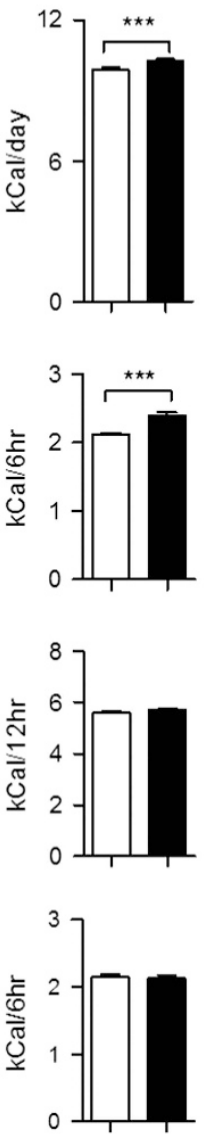
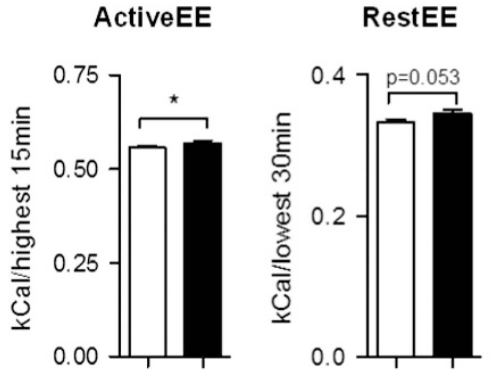

Body Weight
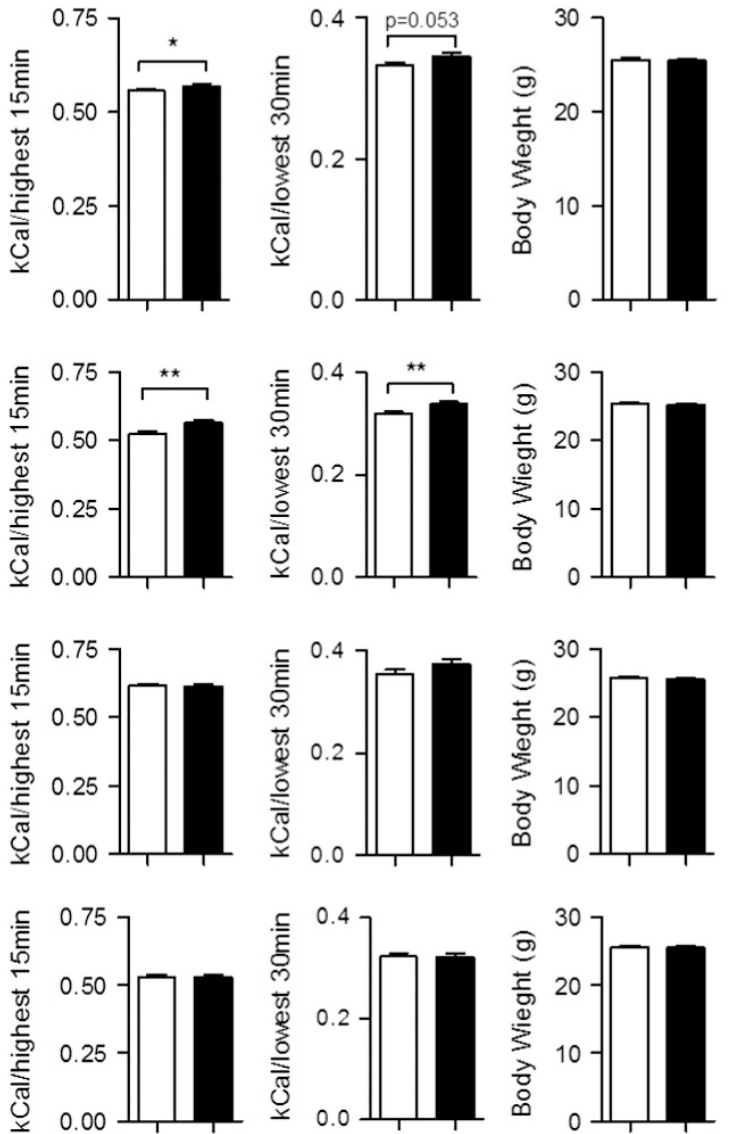

Figure 3. Pharmacosynthetic activation of orexin neurons increases SPA and energy expenditure (EE) in the hours following treatment (male, Cre::hM3Dq, $\mathrm{N}=6$ ). (a) Schematic of treatment and recording periods. Metabolic and behavioral data collected in the home-cage (Sable Promethium; Reno, NV) following CNO $5 \mathrm{mg} / \mathrm{kg}$; black bars) or vehicle (saline; white bars). SPA, TotalEE, ActiveEE, RestEE, and body weight are reported for (b) $24 \mathrm{~h}$ post-injection and divided into (c) LightsON-A (post-injection; $6 \mathrm{hr}$ ), (d) LightsOFF (12 h), and (e) LightsON-B. ${ }^{*} P<0.05$, $* * P<0.01, * * * P<0.001$. 

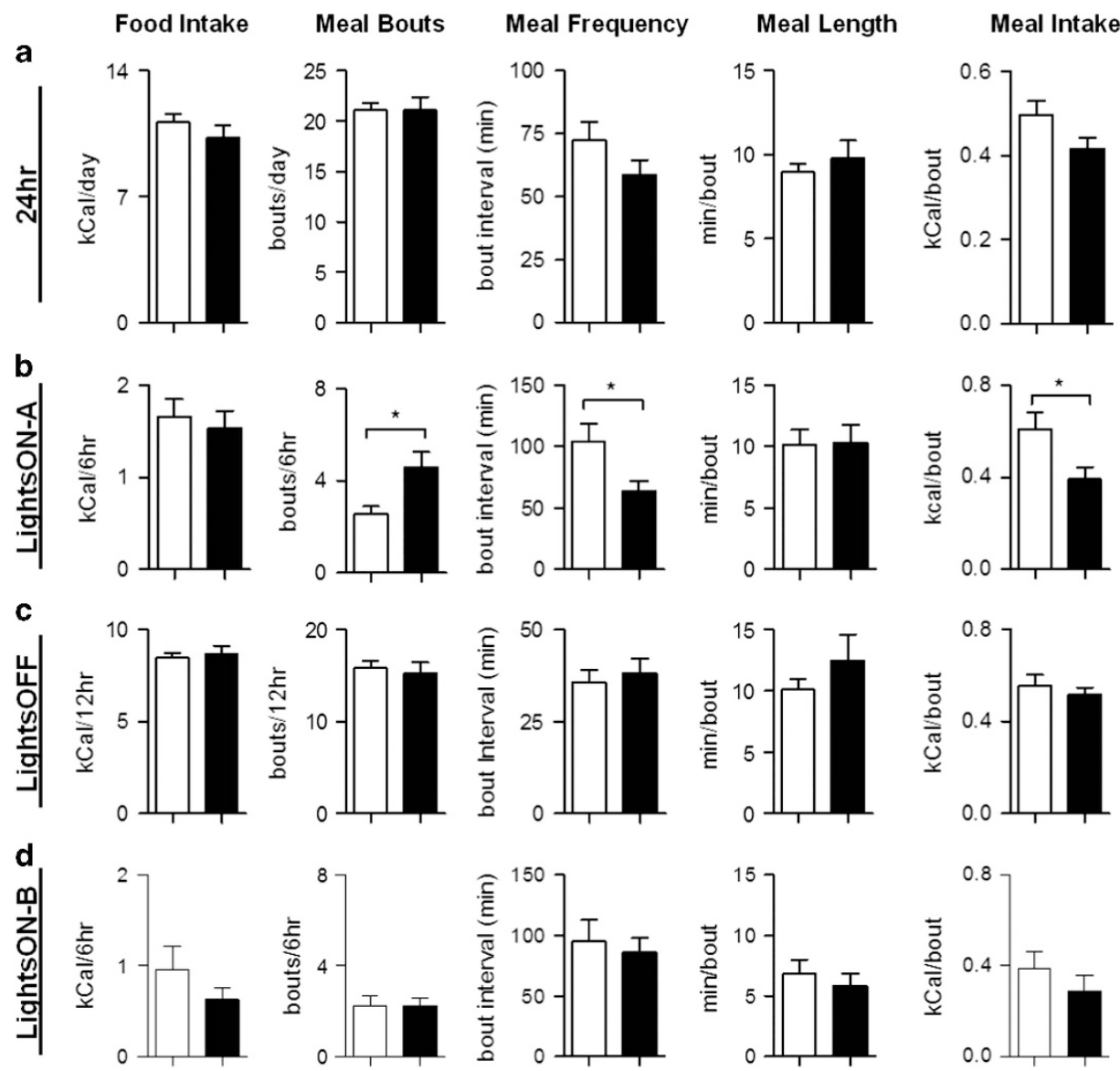

Figure 4. Food (chow) eating behaviors following acute pharmacosynthetic activation of orexin neurons (male, Cre::hM3Dq, $\mathrm{N}=6$ ). Behavioral data collected in the home-cage (Sable Promethium; Reno, NV) following CNO (5 mg/kg; black bars) or vehicle (saline; white bars). Total chow, meals (bouts), bout frequency, bout duration, and bout size are reported for (a) $24 \mathrm{~h}$ post-injection and divided into (b) LightsON-A (postinjection; 6 h), (c) LightsOFF (12 h), and (d) LightsON-B. ${ }^{*} P<0.05,{ }^{* *} P<0.01,{ }^{* * *} P<0.001$.

analysis revealed reduced adiposity levels $10 \mathrm{~d}$ intervention (Figure 5b). DREADD-dependent orexin neuron activation induced greater SPA and EE among mice fed HFD compared to SPA and EE when mice were eating chow (Figures $5 c$ and $d$ ). Clozapine $\mathrm{N}$-oxide did not alter total HFD or water intake (Figures 5 e and $\mathrm{f}$ ). These findings suggest that orexin neuron stimulation is a viable, effective intervention for diet-induced weight gain. In total, these results point to developing orexin-neuron and LH-based therapies for treating overweight and obese conditions in patients.

Changes in SPA, food intake, and EE in female mice following pharmacosynthetic silencing of orexin neurons

To test whether reduced orexin neuron activation would reduce SPA, we used the inhibitory DREADD virus (hM4Di) and evaluated changes in SPA in female mice $(n=6)$. Orexin neuron inhibition reduced SPA in a circadian-dependent manner, with significant reductions observed only during the DarkCycle and not during the LightCycle (Figures $6 \mathrm{a}$ and $\mathrm{b}$ ). Clozapine $\mathrm{N}$-oxide treatments given just prior to LightsOFF decreased SPA in an open-field environment (Figure 6a) and home-cage setting (Figure 6c). Reduced SPA was accompanied by a significant attenuation in EE (Figure $6 \mathrm{~d}$ ). Acute DREADD-dependent inhibition of orexin neurons induced a sustained, reversible effect on SPA and EE (Figure 6). As with excitatory stimulation, hM4Di-induced inhibition treatments had no effect on total calories consumed or gastric motility (fecal boli) (Figures $6 \mathrm{e}$ and $\mathrm{f}$ ). We examined this further by analyzing changes in meal patterns. Compared to the excitation of orexin neurons, there were no significant effects of orexin neuronal inhibition on meal bouts, meal frequency, meal length, or kcal per meal bout (Supplementary Fig. 2A-C).

\section{DISCUSSION}

We used minimally invasive pharmacosynthetic DREADDs to increase or decrease orexin neuron activity in vivo. Experiments uncovered significant differences of sex and time of day on the effects of CNO. Specifically, we observed significant increases in SPA and energy expenditure following orexin neuron activation on both chow and HFD, indicating that orexin neurons are able to increase activity and metabolism during different diet exposures. There were no effects on total food or water intake, during chow or HFD feeding, although within-session meal patterns were slightly altered during excitation of orexin neurons. In addition, the number of fecal boli was unchanged; suggesting overall Gl motility and anxiety-like behaviors were unaffected. ${ }^{21}$ Likewise, inhibition of orexin neurons yielded reductions in SPA and EE, without producing any changes in total food intake or within session meal patterns.

The behavioral and metabolic profile produced by DREADDdependent activation of orexin neurons would be desirable goals for clinical weight loss: sustained elevated SPA and EE without alteration in total food or water consumption. A single, systemic injection of CNO enhanced SPA and EE in orexin-cre mice expressing the excitatory DREADD. Females were more sensitive 
a
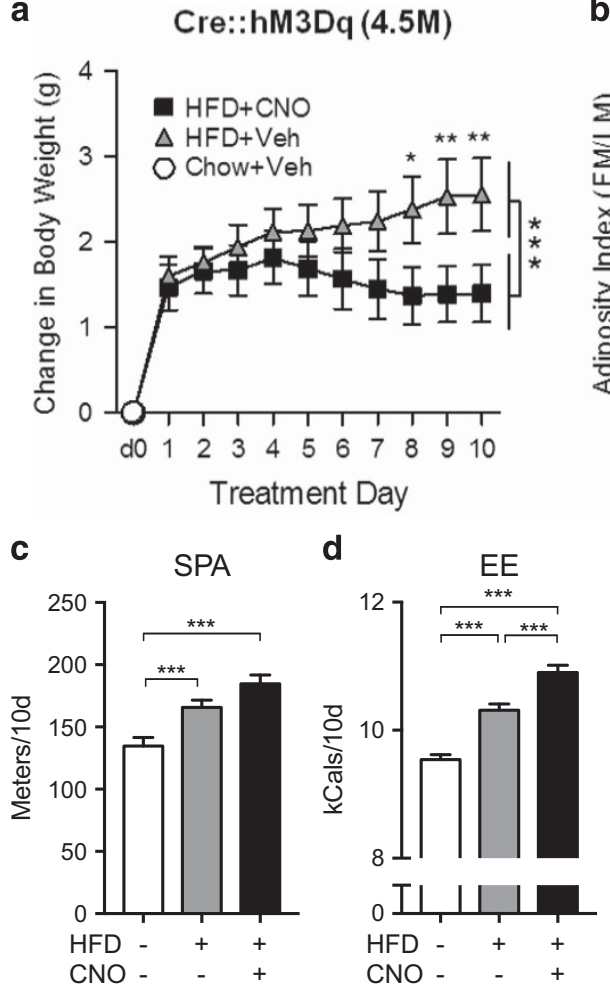

\section{b 10d Intervention}

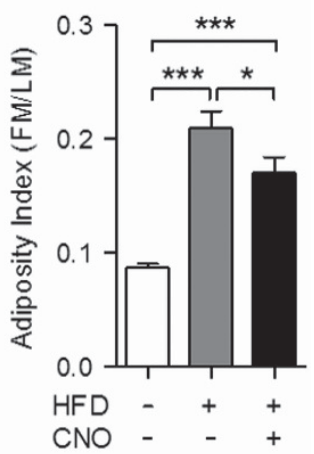

e

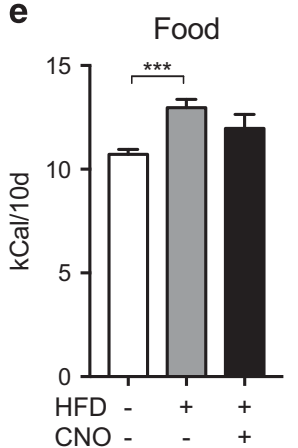

f

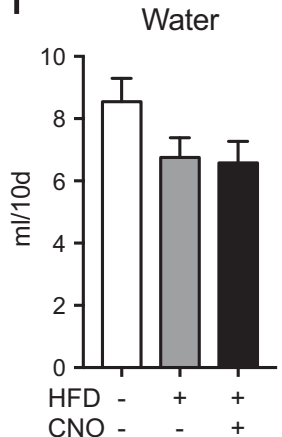

Figure 5. Repeated, daily (10 d) pharmacosynthetic activation of orexin neurons protects against weight gain in adult mice on high fat diet $(\mathrm{N}=14)$. Metabolic and behavioral data collected from adult $(4.5 \mathrm{M})$ male Cre::hM3Dq mice. (a) Weight gain and (b) adiposity (Fat Mass grams/ Lean Mass grams; EchoMRl; Plano, TX) before (d0; Chow+Veh; HFD-/CNO-; white circle or bar) and after $10 \mathrm{~d}$ HFD+CNO (5 mg/kg; black boxes and bars) or HFD+vehicle (veh; saline; grey triangle) treatment. Mean SPA (c), EE (d), food intake (e), and water intake (f) during $10 \mathrm{~d}$ of the Chow+Veh baseline period and the $10 \mathrm{~d}$ periods of HFD with and without CNO*P $<0.05,{ }^{* *} P<0.01,{ }^{* *} P<0.001$.

to acute CNO-dependent activation (Figure 2); which is in line with known sex-differences in orexin neurobiology. ${ }^{22}$ Repeated once daily orexin neuron activation significantly reduced weight gain and adiposity on high fat diet. In the complementary experiment, reducing orexin neuron function by inhibitory DREADD reduced SPA and EE without affecting food or water intake.

Activation of orexin neurons increased SPA at least two-fold in the hours after injection, undoubtedly accounting for a substantial portion of the additional kCals burned. However, kCals burned during periods of inactivity (Resting EE) was also increased; suggesting that other mechanisms for EE were activated in addition to SPA. Thermogenesis from brown adipose tissue activation may be involved, ${ }^{8,23}$ or increased Resting EE could be a carryover effect from SPA and Active EE, akin to post-exercise thermogenesis. Altered patterns of food intake could have resulted in changes in the thermic effects of food that were captured in Resting EE. Most importantly, the single activation of orexin neurons was sufficient to increase total 24-h energy expenditure.

Functional dissociation of neural regulation of SPA, EE, and food intake

Orexin neuron activation increased SPA and EE with a similar magnitude and time course as previous reports. ${ }^{5}$ In contrast, food and water intake effects were strikingly different. Inutsuka and colleagues saw a dramatic increases in chow and water intake, whereas we saw no difference in total consumption of either chow or water. In our studies the pattern of intake was altered in the hours following orexin neuron activation. Animals interacted more often with the food hopper but compensated by reducing the amount consumed per bout. It is possible that the increase in physical activity seen in the present study was driven by an enhanced drive to procure food. However, had this been the case we would have expected to observe a total increase in food intake following CNO injections. Instead, it seems more likely that the increased interactions with the food hopper occurred as a direct result of increased SPA. Unlike the observations of total energy expenditure, short term changes in food intake were offset by complementary reductions in food intake in the later hours. Meal patterns in mice given HFD were not evaluated, but we found no significant changes in total daily food intake in animals on HFD due to CNO, suggesting the pattern of food intake may likely be similar between mice given chow or HFD.

Anatomic targeting may be essential for refining the behavioral output of orexin neuron stimulation. The methods and results reported here differ in key ways from findings published by other groups using orexin excitatory DREADD activation. ${ }^{5,24}$ We opted to inject a moderate volume $(333 \mathrm{nl})$ into a single site per hemisphere aimed at the lateral and caudal extremes of the orexin cell field. The injection site used in this study differed from these studies by an absolute distance of $0.32 \mathrm{~mm}$ in the caudal and lateral direction. ${ }^{5}$ Furthermore, Yamanaka and colleagues $^{5}$ injected twice the volume $(600 \mathrm{nl})$ per injection site, likely producing a larger infection radius, and perhaps resulting in the elevated food intake increase observed in their study. Similarly, Sakurai and colleagues $^{24}$ injected two sites per hemisphere, one in the caudal $\mathrm{LH}$ and another in the rostral LH. In contrast, our studies were purposefully focused only on the orexin neurons in the most lateral aspect of the caudal $\mathrm{LH}$, as these neurons appear to be most important for orexin stimulation of SPA. These differences and others may account for the distinct behavioral and metabolic profiles observed in our studies.

Selective anatomic targeting may also explain the higher dose of $\mathrm{CNO}(5 \mathrm{mg} / \mathrm{kg})$ required to induce robust behavioral 

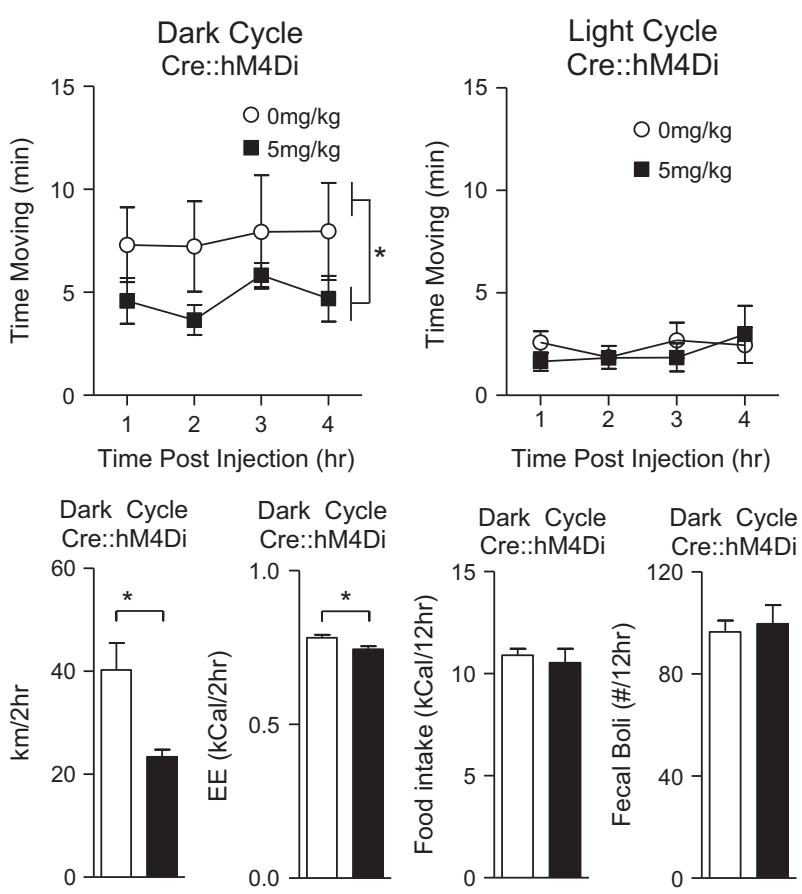

Figure 6. SPA and EE following acute pharmacosynthetic inhibition in Female Cre::hM4Di mice $(n=6)$. Time spent moving in large activity chambers (MedAssociates; St. Albans, VT) $4 \mathrm{~h}$ after an injection of CNO ( $5 \mathrm{mg} / \mathrm{kg}$; black boxes) or vehicle (veh; saline; white circle) and $15 \mathrm{~min}$ before dark cycle onset (a) or $5 \mathrm{~h}$ into the Light Cycle (b); $(\mathrm{N}=4)$. SPA (c) and EE (d) $2 \mathrm{~h}$ post injection in a home-cage environment (Promethion ${ }^{\mathrm{TM}}$; SableSystem, Reno, NV). Total chow intake (e) and fecal boli (f) during the $12 \mathrm{~h}$ dark cycle $(\mathrm{N}=4)$. ${ }^{*} P<0.05,{ }^{*} P<0.01,{ }^{* * *} P<0.001$.

changes. ${ }^{5,24}$ With the higher dose, we induced cFos expression in a similar overall percentage of orexin neurons as groups that delivered $\mathrm{hM} 3 \mathrm{Dq}$ to multiple sites within the $\mathrm{LH}$ or a larger infection radius. ${ }^{5,24}$ The higher dose presumably achieved greater relative activation of lateral $\mathrm{LH}$ orexin neurons clustered in the lateral $\mathrm{LH}$, as well as activation of cells receiving projections from those neurons. However, the potential explanations discussed here will need to be tested empirically.

This study is the first to report significant reductions of SPA and $\mathrm{EE}$ using pharmacogenetic silencing of orexin neurons. We anticipated and observed a pronounced circadian effect. The lack of difference during the LightCycle, a period of low intrinsic orexin activity (Figure 1h), as well as low SPA (Figure 2a), is likely due to a floor effect, such that lower levels of SPA cannot be achieved behaviorally. Alternatively, it is possible that the small amount of basal SPA observed during the LightCycle is driven and regulated by orexin-independent signaling. There was no difference in meal patterns following the orexin neuron inhibition, whereas we did observe changes in meal patterns after orexin neuron stimulation. The difference between studies in meal pattern effects was likely due to the timing differences between the excitatory and inhibitory protocols. CNO was administered halfway through the light cycle in order to facilitate increased SPA when animals normally have low SPA, but CNO was administered at the start of the dark cycle in the inhibitory experiment, to allow inhibition of typical, non CNO-induced, SPA levels during that time of day. In addition, the inhibitory study was conducted in females, as opposed to the males that were used in the excitatory study, with fewer animals per group. Future studies should investigate the short-term effects of orexin inhibition on meal patterns. It is important to again note that no difference in overall food intake per day was seen in either the excitatory or inhibitory studies, suggesting that orexin's most impactful effects on body weight and adiposity are due to changes in physical activity and energy expenditure.

Limitations and other considerations

There are several limitations to the present study and other considerations that should be taken into account. First, there is recent evidence that CNO can produce physiological effects in Long-Evans rats by reducing the acoustic startle reflex and amphetamine-induced hyperlocomotion, but there was no effect of CNO administration on spontaneous locomotor activity, ${ }^{25}$ suggesting its use as an inert compound in the present study is not problematic. A recent publication suggests that effects seen in previous DREADD studies are actually due to the conversion of CNO to clozapine. ${ }^{26}$ That study found that clozapine injections in mice without the active DREADD virus decreased physical activity. However, we did not find any effect of CNO on physical activity in wildtype mice. Previous studies have shown that clozapine reduces physical activity at a dose of $3 \mathrm{mg} / \mathrm{kg}$ and produces no changes in physical activity at $10 \mathrm{mg} / \mathrm{kg}^{27}$ These results would suggest that high levels of clozapine would decrease physical activity. Instead, we saw an increase in physical activity, suggesting that $\mathrm{CNO}$ functioned as theorized by binding to the DREADD receptors to facilitate increases in SPA. Second, while we used both males and females within these studies, varied availability of each sex within the colony resulted in an inability to include comparisons between sexes in some studies. Future studies should directly evaluate orexin neuron activation via DREADDs in both females and males for changes in SPA and EE in the presence of HFD. Third, while we were able to confirm transfection of the virus for the excitation of orexin neurons, we did not investigate this for the inhibition of the orexin neurons. Future studies should determine the rate of transfection for this virus in orexin neurons, although the behavioral data indicate the virus was successfully transfected, as we were able to see a clear decrease in SPA in animals injected with this virus following CNO. Fourth, we stimulated orexin neurons in the lateral LH during periods of lowest physical activity in order to maximize potential effects on SPA. While previous studies of orexin injections into sites of action show that SPA can be elicited even during the dark cycle, $^{3}$ it remains to be tested whether or not orexin neuron stimulation can further enhance SPA when SPA is at its natural peak during the active/dark period.

The research and methodological approaches presented here lay the groundwork for exploring the downstream neural output and molecular mechanisms responsible for the behavioral and physiological changes induced by orexin neuron stimulation. Several genetic tools (i.e., DREADD-constructs with trafficking sequences for axonal or dendritic cell compartments) could be employed to characterize functional orexin neuron populations and parse projection site-specific contributions. For example, a recent study by Kosse et al. 2017 demonstrated that a network of neurons expressing glutamic acid decarboxylase 65 are located within the $\mathrm{LH}$, separate from $\mathrm{MCH}$ and orexin neurons, and may be responsible for facilitating changes in locomotor activity. Specifically, they showed that GAD65 neurons are necessary for carrying out orexin-mediated changes in locomotor activity. ${ }^{28}$ Therefore it is possible that the changes in SPA observed in the present study were facilitated by changes in GAD- 65 produced by orexin neuron activation. It is also possible that the cFos observed in non-orexin neurons within the LH after CNO administration was due to excitation of these neurons.

Our findings suggest that SPA-based interventions work best during times of inactivity. Timing and patterns of sustained inactivity in humans vary widely, as these are intricately woven into the complexity of modern day lifestyles and occupations that beget considerable variation in circadian rhythms. Any adverse effects on 
sleep could potentially be mitigated through timing and dosing, akin to Deep Brain Stimulation tuning in Parkinson's patients. ${ }^{29}$

In summary, SPA is a promising therapeutic target for improving clinical outcomes in obesity and metabolic syndrome. Orexin neurons in the lateral hypothalamus drive SPA and their activity regulates metabolic rate. Stimulation or inhibition of orexin neuron activity via pharmacosynthetic neuromodulation increased or decreased, respectively, SPA and EE. Total food and water intake was unaffected. Repeated, daily stimulation of orexin neurons partially rescues the energy imbalance observed in obesogenic, high-fat diet conditions.

\section{Study approval}

Animal husbandry and experimental protocols were approved by the Institutional Animal Care and Use Committee of the Minneapolis VA Medical Health Care System and were in accordance with the $2013 \mathrm{NIH}$ Guide for the Care and Use of Mammals in Neuroscience and Behavioral Research.

\section{CONFLICT OF INTEREST}

The authors declare no conflict of interest.

\section{ACKNOWLEDGEMENTS}

Sources of Support: This work was supported by the Department of Veterans Affairs (5I01RX000441-04 to CMK and CJB), the National Institute of Health (5R01DK10028103 to CMK and CJB and the Minnesota Obesity Center, 5R01DK100281-03), and by Award Number T32DK083250 from the National Institute of Diabetes and Digestive and Kidney Diseases (NIDDK; to PEB).We are grateful to all of the members of the Obesity Neuroscience group at the Minneapolis VA, especially Martha M. Grace and Morgan R. Little, for their support in establishing the working protocols used within these experiments and productive discussions and constructive comments they provided on the project and manuscript.

\section{AUTHOR CONTRIBUTIONS}

A.N.Z., C.J.B., and C.M.K. conceived and designed the project. A.N.Z., A.A.H., and P.E.B. conducted the experiments and analyzed the data. A.N.Z., P.E.B., C.J.B., and C.M.K. co-wrote the manuscript.

\section{REFERENCES}

1 Buscemi S, Sprini D, Grosso G, Galvano F, Nicolucci A, Lucisano G et al. Impact of lifestyle on metabolic syndrome in apparently healthy people. Eat Weight Disord 2014; 19: 225-232.

2 Hara J, Beuckmann CT, Nambu T, Willie JT, Chemelli RM, Sinton CM et al. Genetic ablation of orexin neurons in mice results in narcolepsy, hypophagia, and obesity. Neuron 2001; 30: 345-354.

3 Kotz CM, Teske JA, Levine JA, Wang CF. Feeding and activity induced by orexin A in the lateral hypothalamus in rats. Regulatory Peptides. 2002; 104: 27-32.

4 Sellayah D, Bharaj P, Sikder D. Orexin Is Required for Brown Adipose Tissue Development, Differentiation, and Function. Cell Metabolism. 2011; 14: 478-490.

5 Inutsuka A, Inui A, Tabuchi S, Tsunematsu T, Lazarus M, Yamanaka A. Concurrent and robust regulation of feeding behaviors and metabolism by orexin neurons. Neuropharmacology, 2014; 85 (c): 451-460.

6 Nishino S, Ripley B, Overeem S, Lammers GJ, Mignot E. Hypocretin (orexin) deficiency in human narcolepsy. The Lancet 2000; 355: 39-40.

7 Schuld A, Hebebrand J, Geller F, Pollmächer T. Increased body-mass index in patients with narcolepsy. The Lancet 2000; 355: 1274-1275.

8 Perez-Leighton CE, Boland K, Billington CJ, Kotz CM. High and low activity rats: Elevated intrinsic physical activity drives resistance to diet-induced obesity in non-bred rats. Obesity 2013; 21: 353-360.

9 Kotz CM, Wang C, Teske JA, Thorpe AJ, Novak CM, Kiwaki K et al. Orexin A mediation of time spent moving in rats: neural mechanisms. Neuroscience 2006; 142: 29-36.

10 Kotz C, Nixon J, Butterick T, Perez-Leighton C, Teske J, Billington C et al. Brain orexin promotes obesity resistance. Annals of the New York Academy of Sciences. Blackwell Publishing Inc 2012; 1264: 72-86.
11 Perez-Leighton CE, Boland K, Teske JA, Billington C, Kotz CM. Behavioral responses to orexin, orexin receptor gene expression, and spontaneous physical activity contribute to individual sensitivity to obesity. AJP: Endocrinology and Metabolism 2012; 303: E865-E874.

12 Peyron C, Tighe DK, van den Pol AN, de Lecea L, Heller HC, Sutcliffe JG et al. Neurons containing hypocretin (orexin) project to multiple neuronal systems. Journal of Neuroscience, 1998; 18: 9996-10015.

13 de Lecea L, Kilduff TS, Peyron C, Gao X, Foye PE, Danielson PE et al. The hypocretins: hypothalamus-specific peptides with neuroexcitatory activity. Proc Natl Acad Sci USA 1998; 95: 322-327.

14 Sakurai T, Amemiya A, Ishii M, Matsuzaki I. Orexins and orexin receptors: a family of hypothalamic neuropeptides and $G$ protein-coupled receptors that regulate feeding behavior. Cell 1998; 92: 573-585.

15 Johren O, Neidert SJ, Kummer M, Dendorfer A, Dominiak P. Prepro-orexin and orexin receptor mRNAs are differentially expressed in peripheral tissues of male and female rats. Endocrinology 2001; 142: 3324-3331.

16 Nakabayashi M, Suzuki T, Takahashi K, Totsune K, Muramatsu Y, Kaneko C et al. Orexin-A expression in human peripheral tissues. Molecular and Cellular Endocrinology 2003; 205: 43-50.

17 Armbruster BN, Li X, Pausch MH, Herlitze S, Roth BL. Evolving the lock to fit the key to create a family of $G$ protein-coupled receptors potently activated by an inert ligand. Proc Natl Acad Sci USA. National Acad Sciences 2007; 104: 5163-5168.

18 Roth BL, Craigo SC, Choudhary MS, Uluer A, Monsma FJ, Shen Y et al. Binding of typical and atypical antipsychotic agents to 5-hydroxytryptamine-6 and 5hydroxytryptamine-7 receptors. Journal of Pharmacology and Experimental Therapeutics. American Society for Pharmacology and Experimental Therapeutics 1994; 268: 1403-1410.

19 Matsuki T, Nomiyama M, Takahira H, Hirashima N, Kunita S, Takahashi S et al. Selective Loss of GABA_B Receptors in Orexin-Producing Neurons Results in Disrupted Sleep/Wakefulness Architecture. Proc Natl Acad Sci USA. National Academy of Sciences 2009; 106: 4459-4464.

20 Bunney PE, Zink AN, Holm AA, Billington CJ, Kotz CM. Orexin activation counteracts decreases in nonexercise activity thermogenesis (NEAT) caused by highfat diet. Physiol Behav 2017; 176: 139-148.

21 Hall CS. Emotional behavior in the rat. I. Defecation and urination as measures of individual differences in emotionality. Journal of Comparative Psychology 1934; 18: 385-403.

22 Brownell SE, Conti B. Age- and gender-specific changes of hypocretin immunopositive neurons in C57BI/6 mice. Neuroscience Letters. 2010; 472: 29-32.

23 Morrison SF, Madden CJ, Tupone D. Central control of brown adipose tissue thermogenesis. Front Endocrinol (Lausanne). Frontiers 2012; 3 [article 5]: 1-5.

24 Sasaki K, Suzuki M, Mieda M, Tsujino N, Roth B, Sakurai T. Pharmacogenetic Modulation of Orexin Neurons Alters Sleep/Wakefulness States in Mice. Manzoni OJ, editor. PLoS One 2011; 6: e20360-e20368.

25 MacLaren DAA, Browne RW, Shaw JK, Radhakrishnan SK, Khare P, España RA et al. Clozapine N-Oxide Administration Produces Behavioral Effects in LongEvans Rats: Implications for Designing DREADD Experiments. Eneuro 2016; 3: 0219-16.

26 Gomez JL, Bonaventura J, Lesniak W. Chemogenetics revealed: DREADD occupancy and activation via converted clozapine. Science, 2017; 357: 503-507.

27 Gururajan A, Taylor DA, Malone DT. Effect of cannabidiol in a MK-801-rodent model of aspects of schizophrenia. Behav Brain Res. 2011; 222: 299-308.

28 Kosse C, Schöne C, Bracey E, Burdakov D. Orexin-driven GAD65 network of the lateral hypothalamus sets physical activity in mice. Proc Natl Acad Sci USA. National Acad Sciences 2017; 114: 4525-4530.

29 Schneider J, Novák D, Jech R. Optimization of Parkinson Disease treatment combining anti-Parkinson drugs and deep brain stimulation using patient diaries, 2015 37th Annual International Conference of the IEEE Engineering in Medicine and Biology Society (EMBC), Milan, 2015, pp. 3444-3447. doi: 10.1109/EMBC.2015.7319133.

(c) (i) $(-)$ This work is licensed under a Creative Commons AttributionBY NC ND NonCommercial-NoDerivs 4.0 International License. The images or other third party material in this article are included in the article's Creative Commons license, unless indicated otherwise in the credit line; if the material is not included under the Creative Commons license, users will need to obtain permission from the license holder to reproduce the material. To view a copy of this license, visit http:// creativecommons.org/licenses/by-nc-nd/4.0/

(c) The Author(s) 2018

Supplementary Information accompanies this paper on International Journal of Obesity website (http://www.nature.com/ijo) 